EPJ Web of Conferences 82, 01025 (2015)

DOI: $10.1051 /$ epjconf/20158201025

(C) Owned by the authors, published by EDP Sciences, 2015

\title{
Simulation of influence of metal fibers on solid burning systems
}

\author{
Boris Borisov $^{1, \mathrm{a}}$, Sergey Bondarchuk ${ }^{2}$ and Alexander Tukaev ${ }^{3}$ \\ ${ }^{1}$ Tomsk Polytechnic University, 634050 Tomsk, Russia \\ ${ }^{2}$ Tomsk State Pedagogical University, 634061 Tomsk, Russia \\ ${ }^{3}$ Engineering Design Bureau, 140402 Kolomna, Russia
}

\begin{abstract}
Considered the process of burning of solid systems with heat-conducting element (HTE). Formulated the mathematical problem of calculating the advancement of the combustion surface along the HTE. The process of warming the solid system is modeled under the integrated approach. Considered the analysis of the results of numerical studies.
\end{abstract}

In the process of designing various power machines, in which is used as Propellant solid systems (SS), to achieve the desired characteristics is often necessary to solve the problem of the maximum possible increase in its rate of combustion. One common solution to this problem is to place in SS of metallic fibers of great length, oriented in the direction of movement of the surface of the SS in the burning process. The metal fibers has a coefficient of thermal conductivity two orders of magnitude greater than the thermal conductivity of the propellant composition. This leads to the fact that the heat from the combustion zone near the surface for SS wire extends deep into solid systems with a higher rate than the burning rate. This heat in turn heats the adjacent layers SS to the fibers until ignition condition shall be achieved. This allowing to increase the rate of the burning front moving along the fiber and forming a conical surface. This idea of the combustion mechanism of SS with heat-conducting element (HCE) is used by all researchers to create models of combustion, allowing to determine the rate of the arrival of the gas mass to combustion chamber. Often these models are focused on getting the final relations for the stationary velocity of the conical surface of the solid systems in the combustion process [1-5]. The obtained relations not too suitable for use in the programs of the calculation of the current geometry of the grain in the combustion process, which is necessary to determine the velocity of the selected point, and not a conical surface. Calculation of non-stationary process is the formation of a conical or other forms of propellant surface as a result of burning in such programs is conducted automatically by a single cross-cutting algorithm. This paper presents a mathematical model that allows to build an efficient algorithm for modeling non-stationary process of the evolution of the combustion surface of the SS, with sufficient accuracy, especially taking into account the effect of advancing the burning surface along the HCE.

In formulating the mathematical model of the combustion burning described above, the following features were taken into account warming up the propellant in the heat-conducting element placement.

\footnotetext{
${ }^{a}$ Corresponding author: bvborisov@tpu.ru
}

This is an Open Access article distributed under the terms of the Creative Commons Attribution License 4.0, which permits unrestricted use, distribution, and reproduction in any medium, provided the original work is properly cited. 
Biot number for the bare part of the fiber diameter $D_{\mathrm{M}}$ built for the heat transfer coefficient $\alpha$ is of minor importance:

$$
\mathrm{Bi}=\frac{\alpha D_{\mathrm{M}}}{\lambda_{\mathrm{M}}} \ll 0.1
$$

The relation between the coefficients of thermal conductivity of the propellant and metal $\lambda_{\mathrm{T}} \lambda_{\mathrm{M}}$, as noted above, has approximately the same order of magnitude as the Biot number:

$$
\frac{\lambda_{\mathrm{T}}}{\lambda_{\mathrm{M}}} \ll 0.1 \text {. }
$$

For these relations fiber can be regarded as thermally thin body, and in this regard heating wire along its length $\mathrm{x}$ simulated one-dimensional heat equation, written for an arbitrary elementary volume:

$$
V \frac{\partial T}{\partial t}=a S \frac{\partial^{2} T}{\partial x^{2}}-\frac{F}{c \rho} q .
$$

In this equation, $V, S, F$ - volume, cross sectional area and the area of the lateral surface of the cylindrical fiber; $a$ - thermal diffusivity of the metal fiber; $q$ - heat flux through the lateral surface, which is determined for the bare part of the wire in accordance with law of Newton and Richman:

$$
q=\mathrm{a}\left(T-T_{\text {burning }}\right),
$$

but what about fiber inside the propellant - the law of the Fourier heat conduction, in accordance with the temperature gradient in the propellant on the side surface of the fiber:

$$
q=-\left.\lambda_{\mathrm{T}} \frac{\partial T}{\partial r}\right|_{r=r_{\mathrm{M}}}
$$

The above analysis, confirmed by results of two-dimensional calculations, shows that the warming up propellant depth is several orders of ( 2 to 4 ) is less than the warming up fiber depth. This makes it possible to simulate the heating of the propellant and to determine the heat flow at the side surface of the wire within the integrated approach.

This approach is based on specifying the temperature profile as a known function and replaced the Partial differential equation by ordinary differential equations.

After the analysis as a function defining a temperature profile in the propellant $T_{\mathrm{T}}$ for the selected area of the solution (Fig. 1), after the conducted numerical study was chosen cubic parabola for values $\theta=T_{\mathrm{T}}-T_{0}$ relatively $\xi=r-r_{\mathrm{M}}$ where $T_{0}$ initial temperature of the propellant:

$$
\theta=\theta_{\mathrm{M}}\left[1-\frac{\xi}{\delta}\right]^{3} .
$$

This temperature profile propellant meets the conditions of equality of temperature on the surface of the metal wire and the smooth achievement of the values of the initial temperature at the depth of the warm $T_{0} \xi=\delta$ :

$$
\begin{gathered}
\left.\theta\right|_{r=r_{\mathrm{M}}}=\theta_{\mathrm{M}}=T-T_{0}, \\
\left.\theta\right|_{\xi=\delta}=0,\left.\frac{\partial \theta}{\partial \xi}\right|_{\xi=\delta}=0,\left.\frac{\partial^{2} \mathrm{~h}}{\partial \xi^{2}}\right|_{\xi=\delta}=0 .
\end{gathered}
$$

In accordance with the selected profile on the border wire temperature gradient is determined by the relation:

$$
\left.\frac{\partial \theta}{\partial \xi}\right|_{\xi=0}=-\frac{3 \theta_{\mathrm{M}}}{\delta} .
$$




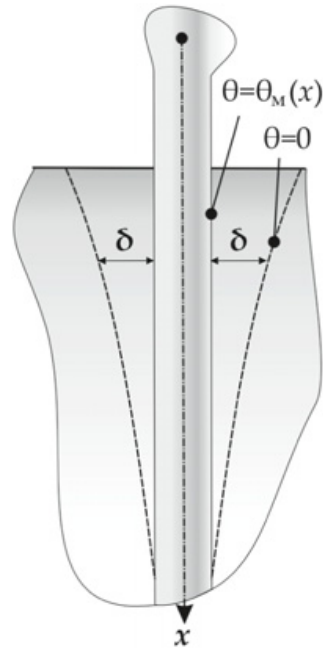

Figure 1. Schematic field solutions.

Depth of heating propellant during the time t corresponds to the heat stored in the propellant:

$$
Q=\int_{0}^{t}-\left.\lambda_{\mathrm{T}} \frac{\partial \theta}{\partial \xi}\right|_{\xi=0} d t=\int_{0}^{t} \lambda_{\mathrm{T}} \frac{3 \theta_{\mathrm{M}}}{\delta} d t .
$$

On the other hand, this heat is determined by the integrate with using the resulting temperature profile in warm by propellant depth, assuming a constant value of the specific heat $\left(c_{\mathrm{T}}\right)$ and the density of the propellant $\left(\rho_{\mathrm{T}}\right)$ :

$$
Q=c_{\mathrm{T}} \rho_{\mathrm{T}} \int_{0}^{\delta} 2 \pi\left(\xi+r_{\mathrm{M}}\right) \theta_{\mathrm{M}}\left[1-\frac{\xi}{\delta}\right]^{3} d \xi .
$$

After this transformation the integral is written as follows:

$$
Q=2 \pi c_{\mathrm{T}} \rho_{\mathrm{T}} \theta_{\mathrm{M}}\left[\frac{r_{\mathrm{M}} \delta}{4}+\frac{\delta^{2}}{20}\right] .
$$

The resulting quadratic equation with respect to the depth of heating is permitted and after analyzing the roots value $\delta$ is determined according to the following relationship:

$$
\delta=2.5 r_{\mathrm{M}}\left[\sqrt{1+\frac{Q}{12.5 \pi c_{\mathrm{T}} \rho_{\mathrm{T}} \theta_{\mathrm{M}} r_{\mathrm{M}}^{2}}}-1\right] .
$$

During calculation, at each time step, the current value of the temperature gradient at the boundary wirepropellant (1) uses for a numerical integration of (2) to determine the current value of the stored heat in the propellant and after it to the altered depth value is calculated in accordance with the heating (3).

Using this approach, calculations were carried out several model variants, the respective calculations, the results of which are given in [1-5]. Satisfactory agreement in the values of the stationary velocity of the combustion surface along the wire was achieved. It should be noted that as the criterion time ignition takes the moment when the temperature reaches the critical value of the propellant, which is selected from the range from $500^{\circ} \mathrm{S}$ to $600^{\circ} \mathrm{S}$. The length value of the bare portion of the wire is determined by the process of promoting the burning surface of the SS and the melting point of the metal, followed by blows his flow of combustion products. In papers [1-5] recommended the heat transfer coefficient 
from the combustion zone to determine from the consideration of the products of combustion of SS flow in the conical surface grain around the stripped portion of the wire. This approach does not fully comply with the burning of metallized propellants, in which at low flow velocities of the combustion products at the surface of SS becomes the main mechanism of heat transfer radiative heat transfer. It is proposed to use the heat transfer coefficient as an external parameter determined in the process of finding the basic parameters of the combustion chamber. Varying heat transfer coefficients in the range from $10 \mathrm{~W} /\left(\mathrm{m}^{2} \mathrm{~K}\right)$ to $500 \mathrm{~W} /\left(\mathrm{m}^{2} \mathrm{~K}\right)$ leads in the case of steel wire $D_{\mathrm{M}}=2 \mathrm{~mm}$ for the maximum change value of the thickness $\delta$ of from 50 microns to 10 microns, wherein the rate of advance of the stationary surface of the combustion of propellant along the wire exceeds the burning rate, respectively, from 2 to 7 times. What is in satisfactory agreement with the data of natural experiments.

\section{References}

[1] G. Golub Journal of Spacecraft and Rokets, 1965, 2, No 4, 593-594

[2] L.H.Caveny, R.L. Glick. Journal of Spacecraft and Rockets. 4, Jan. 1967. Pp. 79-85

[3] N.N. Bahman, I. N. Lobanov. Combustion, Explosion and Shock Waves. Novosibirsk, Russia, 1975

[4] Cubota N., Ichida M., Fujisawa T. AIAA Journal 20.1 (1981): 116-122. Print

[5] N.N. Bahman, I.N. Lobanov. Combustion, Explosion and Shock Waves. Novosibirsk, Russia, 1983 